\title{
Information processing under versus under punishment
}

\author{
Timothy Ballard, David K. Sewell, Daniel Cosgrove, \& Andrew Neal \\ The University of Queensland
}

\begin{abstract}
Much is known about the effects of reward and punishment on behavior, yet little research has considered how these incentives influence the information processing dynamics that underlie decision making. We fit the linear ballistic accumulator model to data from a perceptual judgment task to examine the impacts of rewardand punishment-based incentives on three distinct components of information processing: the quality of the information processed, the quantity of that information, and the decision threshold. The threat of punishment lowered the average quality and quantity of information processed compared to the prospect of reward or no performance incentive at all. The threat of punishment also induced less cautious decision making by lowering people's decision thresholds relative to the prospect of reward. These findings suggest that information processing dynamics are not wholly determined by objective properties of the decision environment, but also by the higher order goals of the system.

Keywords: Information Processing | Reward | Punishment | Decision Making | Computational Modeling
\end{abstract}

\section{Information processing in the face of reward versus punishment}

One of the most fundamental drivers of human behavior is our motivation to obtain rewards and avoid punishment. Sensitivity to pleasure and pain has been critical for our survival (Leknes \& Tracey, 2008). Animals that could successfully respond to rewarding stimuli (e.g., mates) and evade threatening stimuli (e.g., predators) stood a greater chance of passing on their genes. Motivation for seeking reward and avoiding punishment may also be critical for shaping decision-making on a more moment-to-moment basis, as people respond differently to situations in which they are rewarded for success versus punished for failure (Frank, Seeberger, \& O'Reilly, 2004).

Whilst much is known about how reward and punishment influence motivation and behavior, less is known about their effects on decision making. Contemporary models of decision making assume that people process information by integrating information sampled from the environment

T. B. and D. K. S. were supported by ARC Discovery Early Career Researcher Awards (DE180101340 and DE140100772 respectively). A. N. was supported by an ARC Discovery Project (DP150102658). We thank Paul Jackson for programming the experiment, and Hector Palada and Andrew Heathcote for their advice on the computational modeling. 
over time until it provides sufficient evidence for an action (Brown \& Heathcote, 2008; Ratcliff \& Smith, 2004; Sewell \& Smith, 2016). In this research, we consider the effects of reward and punishment on three components of information processing: 1) the average quantity of task-relevant information that is sampled in a given unit of time, 2) the quality or diagnostic value of task-relevant information that is sampled, and 3) and the decision threshold that determines when to act on that information.

We use a well-validated computational model-the Linear Ballistic Accumulator (LBA)-to quantify these components of the information processing system. The LBA (Brown \& Heathcote, 2008) assumes that evidence is accumulated for each response alternative independently until the evidence for one alternative breaches a threshold, at which point that alternative is selected. The LBA estimates a mean drift rate parameter for each response alternative, which summarizes the amount of evidence accumulated for that alternative per unit of time. The difference in the mean drift rates between the correct and incorrect alternatives summarizes the discriminative evidence driving the decision process, capturing the quality of the information being processed. A novel aspect of this research is our use of the LBA to measure the quantity of information processed, which can be indexed by taking the sum of the drift rates for the correct and incorrect responses. The LBA also estimates a threshold parameter for each response alternative, which quantifies the amount of evidence required to select that alternative, and reflects the cautiousness of the decision maker. By examining how these parameters are affected by reward and punishment, we can make inferences about how these factors might influence the information processing dynamics that underlie decision making.

There are a number of ways that reward and punishment might influence information processing. One possibility is that people may invest more effort when seeking reward or avoiding punishment (Brehm \& Self, 1989; Kruglanski et al., 2012). Many have argued that the application of mental effort is costly, and that people will only exert effort if the benefits outweigh the costs (e.g., Kahneman, 1973; Kool \& Botvinick, 2014). The effect of increasing the amount of effort that is allocated to a task is to enhance the information processing capacity for that task.

Additional processing capacity could influence the decision process in multiple ways. First, it might improve the ability of the decision maker to discriminate between competing response alternatives, which would correspond to an increase in the quality of the information extracted from the environment. Second, it might enable the decision maker to process information more rapidly, without necessarily affecting the ability to discriminate between alternatives. This would correspond to an increase in the quantity of the information that is processed per unit time. The quality and quantity of information processing are theoretically distinct. Yet studies that have used sequential sampling models like the LBA to examine the mechanisms underlying decision making have focused almost exclusively on the quality component, with no research (to our knowledge) explicitly disentangling the two constructs. Thus, it is unclear whether reward and punishment should affect quality, quantity, both, or neither.

A competing perspective is that the presence of reward or punishment may induce performance monitoring, which diverts resources away from the focal task (e.g., Hockey, 1997; Kanfer \& Ackerman, 1989). For example, Kanfer and Ackerman (1989) argue that when people are given a goal to achieve, they actively monitor their performance, and that this can impair performance because it consumes resources. Under this view, reward and punishment may hinder the ability to process task-related information if the performance monitoring costs cannot be offset by increases in processing capacity. This account makes competing predictions to the capacity account described 
above. These arguments suggest that reward and punishment may decrease the amount of resources that are available for the task itself, which could reduce the quality and/or quantity of the information that is extracted from the environment.

There is also reason to believe that the effects of punishment may be different to the effects of reward. Research on loss aversion suggests that people are more motivated to avoid losses than to obtain gains of equivalent magnitude (Kahneman \& Tversky, 1979). This may lead people to apply more effort when faced with punishment compared with rewards, leading to enhanced capacity under punishment. In this case, the quality and/or quantity of information processing should be greater under punishment than reward. However, loss aversion might also increase the tendency to monitor one's performance when under threat of punishment, which would detract from the resources available for the task itself. In this case, the quality and/or quantity of information processing should be greater under reward than punishment. It is also possible that both of these effects emerge, in which case the difference in quality and quantity between reward and punishment would depend on the extent to which increases in capacity due to effort are offset by performance monitoring costs.

Finally, it is possible that reward and punishment influence not only the quality or quantity of information processing, but also the threshold that determines the amount of information required before an action is enacted. Regulatory focus theory (Higgins, 1997) proposes that people are more cautious when focused on preventing negative outcomes compared to when pursuing positive outcomes. This suggests an asymmetry in the amount of information people process before being willing to commit to a course of action. Specifically, regulatory focus theory suggests that people may require less information, and thus set a lower response threshold, when seeking reward than when avoiding punishment.

In the following sections, we report an experiment that examines these processes using a well-established perceptual judgment paradigm. We manipulate reward and punishment within participants, and use the LBA to measure quality and quantity of information processed, and the decision threshold in each condition.

\section{Method}

Thirty-five undergraduate students from the University of Queensland (23 females, 12 males, mean age $=19.89)$ performed a random dot motion discrimination task. In each trial, participants were presented with a cloud of 40 moving dots (see Figure 1). Across successive frames during stimulus presentation, some proportion of the dots moved coherently either toward the top-left or the top-right of the cloud, whilst the other dots were moved randomly. The participant's task was to identify whether the dots were moving mostly to the left or mostly to the right. Difficulty was manipulated by varying the proportion of the dots moving coherently $(0.08=$ Easy, $0.06=$ Medium, $0.05=$ Hard $)$.

The stimulus was generated using a version of the "white noise" algorithm (Pilly \& Seitz, 2009). The diameter of the cloud stimulus was approximately $33 \%$ of the screen height. Each dot had a diameter of approximately $0.66 \%$ of the screen height. The dot positions were updated approximately every $66.6 \mathrm{~ms}$. On each update, dots were independently selected according to the trial coherence level to move coherently or randomly on the next update. Coherent dots moved at a rate such that it would take a single dot $3000 \mathrm{~ms}$ to traverse the entire cloud. If the new location would be outside the cloud, the dot was repositioned randomly. Non-coherent dots were repositioned randomly. 


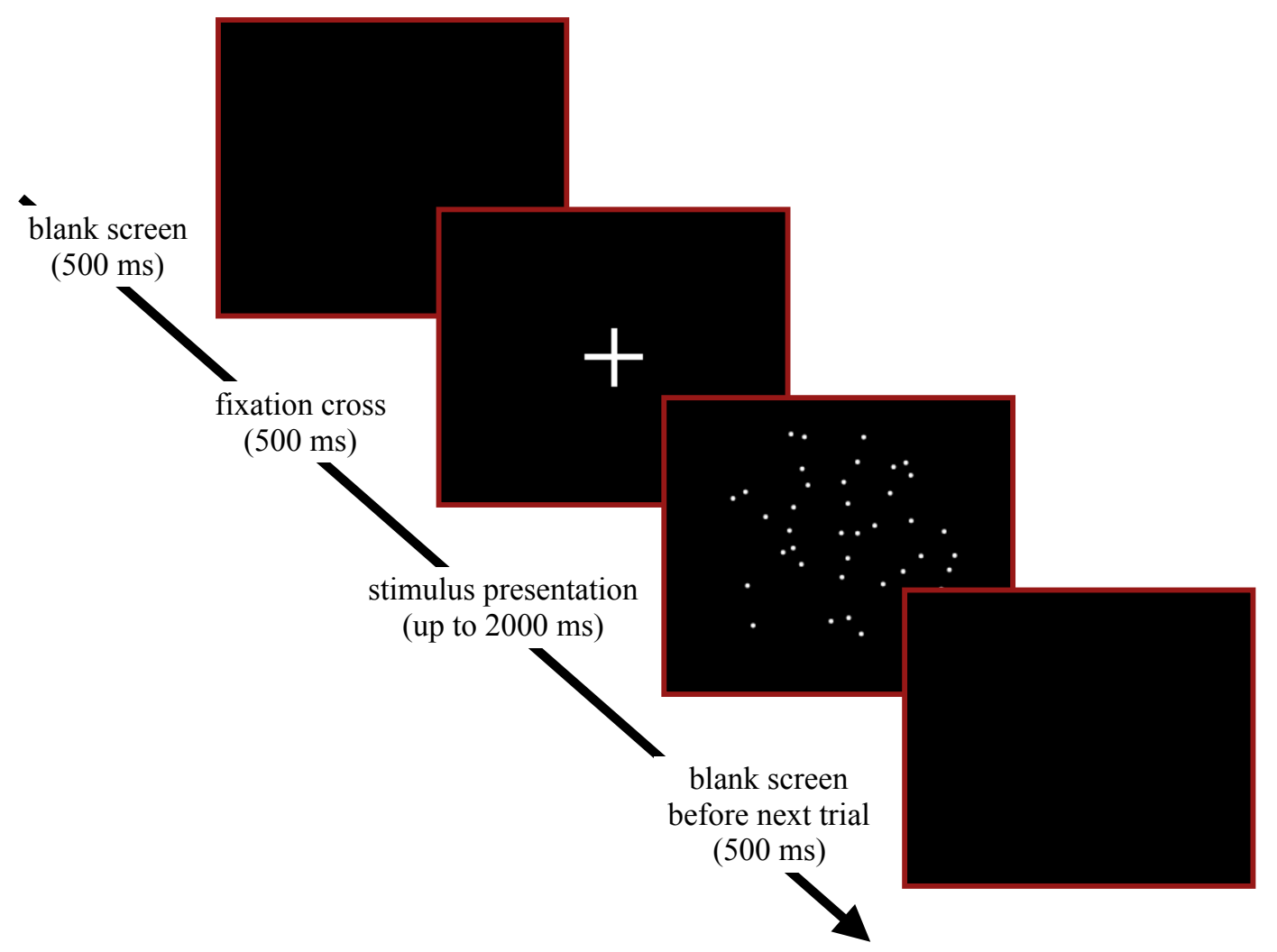

Figure 1. Sequence of an experimental trial. Not that the stimulus image is not drawn to scale.

At the start of the experiment, participants were instructed to press the "z" key if the dots were streaming up to the left and the " $p$ " key if they were streaming up to the right. They then completed 10 practice trials. The experiment was broken down into 9 blocks of 90 trials each. For each block, participants were given the goal of achieving $77 \%$ accuracy and a mean response time of less than 1.071 seconds (these values were based on the accuracy and mean response time observed in a pilot study). Participants started the experiment with an endowment of \$7.50 (AUD). Reward and punishment were manipulated within participants by varying the monetary incentive associated with the block (see Galea, Mallia, Rothwell, \& Diedrichsen, 2015; Guitart-Masip et al., 2012; O’Doherty, Kringlebach, Rolls, Hornak, \& Andrews, 2001; Palminteri, Khamassi, Joffily, \& Coricelli, 2015, for similar manipulations). In one third of blocks, participant gained $\$ 2.50$ when they achieved the goal (the reward condition). In another third, participants lost $\$ 2.50$ when they did not achieve the goal (and did not gain any money when they achieved it; the punishment condition). In the other third, they could not gain or lose any money regardless of whether or not they achieved the goal (the neutral condition).

The block incentives occurred in random order, but were stratified such that each condition occurred once in blocks 1-3, once in blocks 4-6, and once in blocks 7-9. At the start of each block, participants were reminded of the goal and were told the consequences of achieving or failing it in that block. Within each block, half of the stimuli displayed dots moving up and to the left and the 
other half displayed dots moving up and to the right. Difficulty was also manipulated within blocks by varying the level of coherent motion across trials. The order of these stimuli was randomized. Each trial terminated when the participant made a response or after 2 seconds, whichever came first. If the participant did not make a response within 2 seconds, a "TOO SLOW" message appeared and the trial was recorded as a non-response. At the end of the block, the participant received feedback regarding whether or not they had achieved their block goal. However, participants did not receive trial-by-trial feedback regarding the correctness of each decision.

The resulting sample size was 29,160 total experimental trials (35 participants $\times 810$ trials each). This is consistent with the recommended sample size for accurate parameter estimation using the LBA (e.g., Donkin, Averell, Brown, \& Heathcote, 2009). We excluded from the analysis all practice trials, non-responses, and trials in which the observed response time was $250 \mathrm{~ms}$ or less. These response times are so short that the participant could not have plausibly made a decision during this time. The excluded cases made up just over $3 \%$ of all trials. The data and code necessary to conduct the analyses, as well as the source code used to generate the experimental stimuli, are publicly available, and can be found at https://osf.io/jhprb.

\section{Results}

We first considered the effects of the experimental manipulations on accuracy and response time (see Figure 2). We analyzed these effects using Bayesian methods (full details regarding the analyses are included in the supporting information). We report the Bayes factor (BF) as a measure of the evidence for each effect, using the heuristic classification scheme reported by Lee and Wagenmakers (2013) to describe the strength of evidence. We also report the 95\% credible interval on the estimate in order to evaluate the magnitude of each effect.

When people were rewarded for goal achievement, we found "moderate" evidence for no change in accuracy when compared with the neutral (i.e., no-incentive) condition $(B F=0.01, C I$ $=-0.03,0.10)$. We also found "anecdotal" evidence for no difference in response time between the reward and neutral conditions $(B F=0.39, C I=-7.30,9.09)$. When participants were punished for goal failure, we found "extreme" evidence for a difference in accuracy relative to the neutral condition, with accuracy being lower under punishment $(B F=184.06, C I=-0.24,-0.11)$. We also found "moderate" evidence for a difference in response time between the punishment and neutral conditions, with participants taking longer to respond under punishment $(B F=4.09, C I=1.13$, 18.58). We found "very strong" evidence for an effect of difficulty on accuracy, with participants responding less accurately as difficulty increased $(B F=92.77, C I=-0.20,-0.14)$. We also found "extreme" evidence for an effect of difficulty on response time, with participants taking longer to respond as difficulty increased $(B F=9076.23, C I=15.49,25.25)$.

As can be seen in Figure 2, when participants were threatened with punishment, they responded about as accurately (and no faster) to easy stimuli as they did to medium difficulty stimuli when either rewarded or not incentivized. Likewise, they responded about as accurately (and no faster) to medium difficulty stimuli under punishment as they did to hard stimuli under reward. These results point to a considerable performance cost associated with the threat of punishment.

Whilst the behavioral results are suggestive, we cannot make inferences about the underlying decision process based on these results alone. Specifically, we cannot determine the extent to which the effects on accuracy and response times are due to changes in the quality of information people process under different incentives, the quantity of information being processed, or to changes in decision thresholds. To examine the underlying information processing dynamics, we fit the choice 


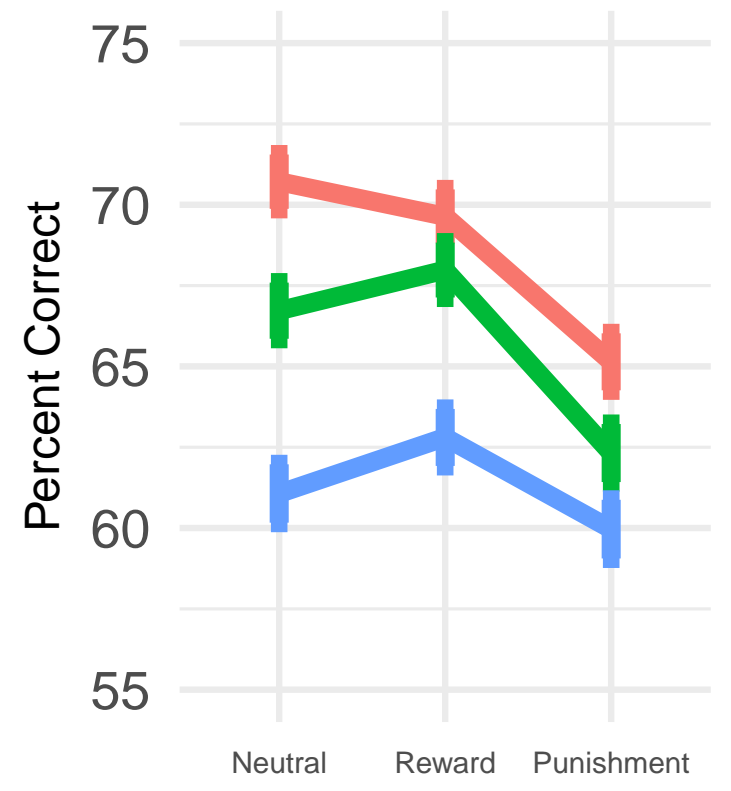

\section{Difficulty}

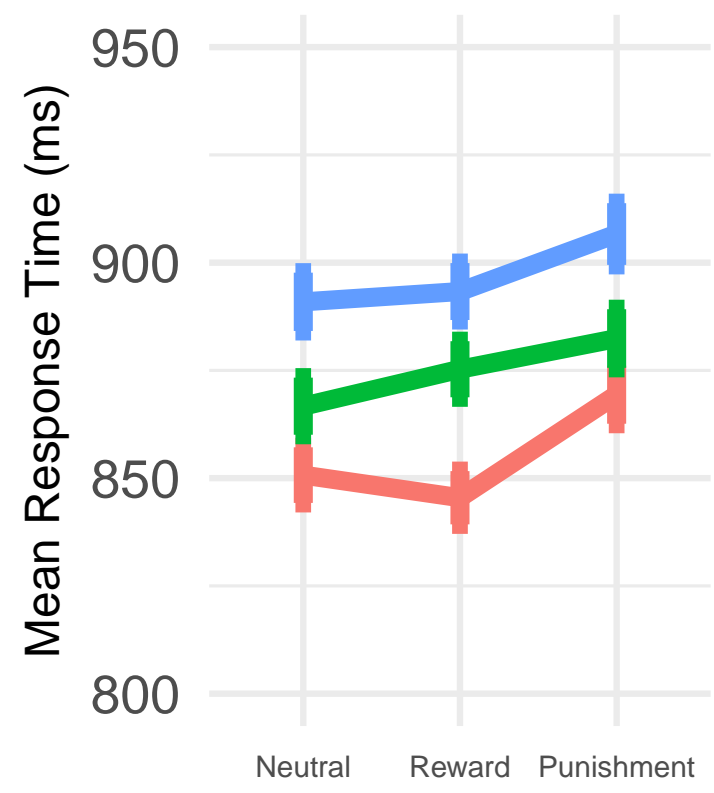

Figure 2. Proportion correct and mean response time (left and right panels respectively) as a function of difficulty in the neutral, reward, and punishment conditions. The error bars indicate the standard error around each value.

and response time data using the LBA model within a hierarchical Bayesian framework. We report the results of this analysis in the next section.

\section{Quality and Quantity of Information Processed}

Using the measures described above, we first examined the quality and quantity of the information being processed in the reward, punishment, and neutral conditions (see Figure 3). We found "extreme" evidence for a difference in the quality of information processed, which reflects the ability to discriminate between rightward and leftward motion, between the reward and punishment conditions. Quality was higher in the reward condition than in the punishment condition $(B F$ $=15423, C I=0.18,0.32$ ). We also found "extreme" evidence for a difference in the quality of information processed between the punishment and neutral conditions, with the quality being higher in the neutral condition than in the punishment condition $(B F=15466, C I=0.13,0.27)$. Finally, we found "moderate" evidence for no difference in the quality of information processed between the reward and neutral conditions $(B F=0.19, C I=-0.03,0.11)$.

With regard to the quantity of information processed, we found "extreme" evidence for a difference between the reward and punishment conditions, such that, overall, participants processed more information in the reward condition than in the punishment condition $(B F=22973, C I=0.30$, 0.51 ). We also found "extreme" evidence for a difference in the quantity of information processed between the punishment and neutral conditions, with quantity being higher in the neutral condition 
than in the punishment condition $(B F=22003, C I=0.20,0.40)$. Finally, we found "anecdotal" evidence for no difference in the quantity of information processed between the reward and neutral conditions $(B F=0.74, C I=-0.01,0.20)$.
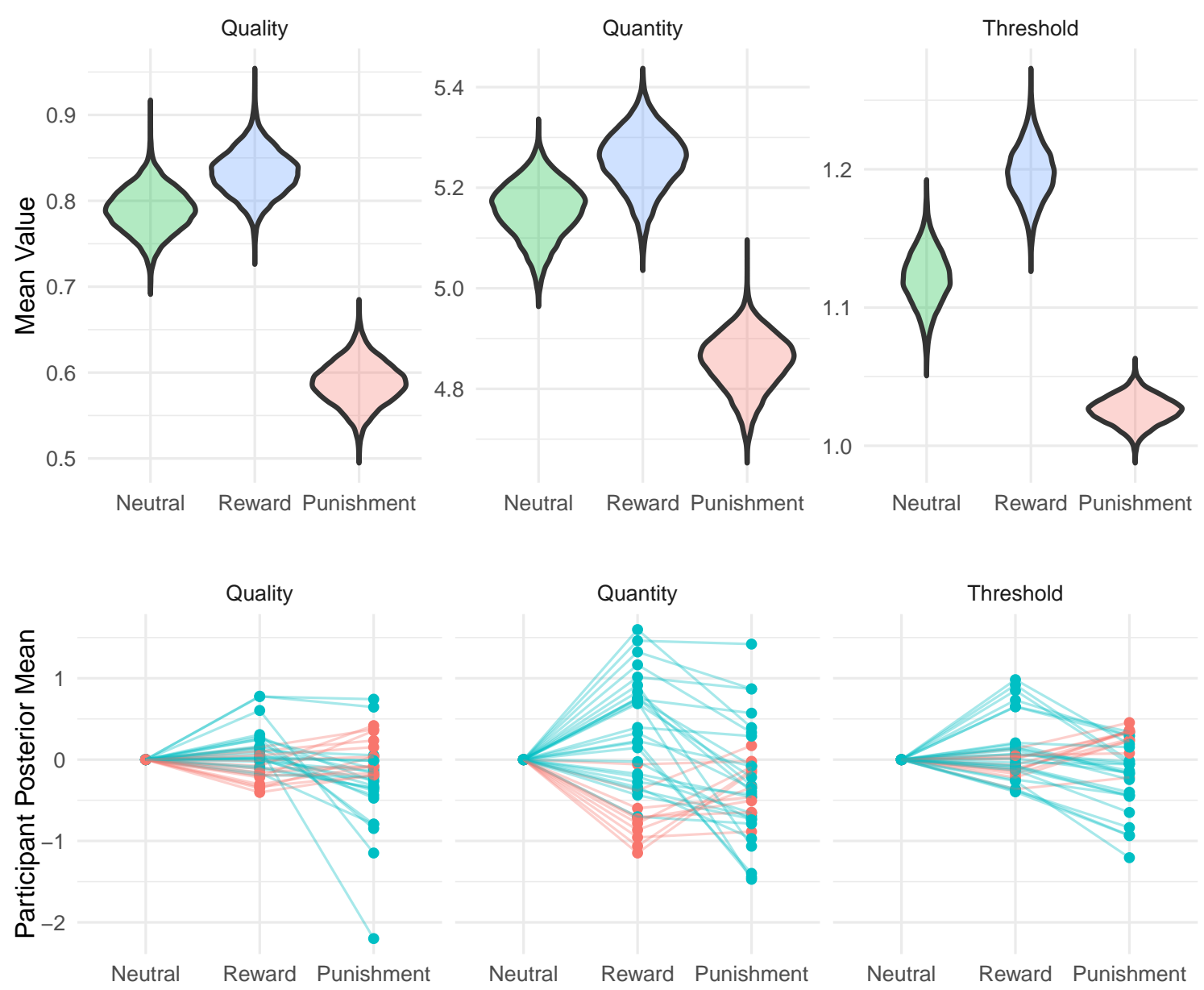

Figure 3. Quality and quantity of information processing, and the decision threshold (left, middle, and right columns respectively) in neutral, reward, and punishment conditions. Quality was determined by the difference in mean drift rates. Quantity was determined by the sum of the mean drift rates. Quality and quantity were collapsed across difficulty. The decision threshold was collapsed across response. The violin plots in the top row represent the posterior density of the condition mean. The bottom row displays posterior means for each participant, which were normalized by subtracting the value in each condition from the corresponding value in the neutral condition. The blue lines in the bottom row indicate participants for whom the relevant component has a higher value in the reward condition than in the punishment condition. The red lines indicate participants for whom the component has a higher value in the punishment condition than in the reward condition.

The results reported above suggest that, on average, both the quality and quantity of information processed was lower under punishments than it was under rewards or in the neutral condition. 
The bottom row of Figure 3 breaks these effects down at the participant level. In each panel, participants for whom the relevant component had a higher value under rewards than under punishments are shown in blue, whereas participants for whom the component had a higher value under punishments than rewards are shown in red. As can be seen, the quality of the information processed was lower under punishments than under rewards for 22 of the 35 participants $(63 \%)$, and quantity was lower under punishments than under rewards for 25 participants (71\%). Quality was lower under punishment than in the neutral condition for 25 participants $(71 \%)$, whereas quantity was lower under under punishment than in the neutral condition for 27 participants (77\%). Quality was higher under reward compared to the neutral condition for 20 participants (57\%), whereas quantity was higher under reward compared to the neutral condition for 17 participants (49\%).

\section{Decision Thresholds}

We next examined the amount of evidence required to make a decision in each condition. Our analysis yielded "extreme" evidence for differences in the average decision thresholds, which reflect the amount of evidence required to make a decision, across all three conditions (see top right panel of Figure 3). On average, thresholds were higher under rewards than punishments $(B F=20008, C I$ $=0.14,0.21)$, higher in the neutral condition than in the punishment condition $(B F=20114, C I=$ $0.07,0.13)$, and higher in the reward condition than in the neutral condition $(B F=2145, C I=0.04$, $0.11)$.

As can be seen in the lower right panel of Figure 3, threshold was lower under punishment than under reward for 26 out of 35 participants (74\%). Threshold was lower under punishment than in the neutral condition for 20 participants (57\%). Finally, threshold was higher under reward than in the neutral condition for 18 participants $(51 \%)$.

\section{Discussion}

We examined the impact of reward and punishment on the information processing that underlies perceptual decision making. We found that people responded more slowly and less accurately when threatened with punishment than when offered a reward or when not incentivized. We decomposed these effects by using the Linear Ballistic Accumulator model (Brown \& Heathcote, 2008) to measure the quality and quantity of information processing, and the decision thresholds that people set. Our findings suggest the presence of reward did not systematically change the quality of information processed (i.e., the discriminative value of the evidence) or the quantity of information processed (i.e., the total amount of evidence that can be processed per unit of time), relative to the neutral condition. The simplest explanation for this finding is that reward neither increases overall information processing capacity nor induces performance monitoring behaviors that divert resources away from the task. However, it is also possible that reward has both of these effects, but that the increase in capacity is offset by the performance monitoring cost.

Punishment on average reduced both the quality and quantity of information processed relative to the reward and neutral conditions. This result suggests that the threat of punishment compromises the ability to process task-related information. This finding is consistent with the notion that, when people expect to be punished for goal failure, they may be more likely to engage in performance monitoring behaviors that reduce the resources available for task performance (Hockey, 1997; Kanfer \& Ackerman, 1989). However, a question remains as to why the performance cost is incurred under punishment and not reward. One potential explanation is that loss aversion makes 
the punishment more salient than the reward, which makes people more likely to engage in costly performance monitoring in the former context. An alternative explanation is that reward and punishment promote performance monitoring to the same extent, but only reward increases processing capacity enough to compensate for the monitoring costs. Future work is needed to disentangle these competing explanations, for example, by directly measuring the extent to which people monitor their performance.

The amount of evidence people required before making a decision also differed across conditions. On average, people set higher thresholds under reward than under punishment, suggesting that people use more cautious strategies when offered a reward than when threatened with punishment. This result runs counter to regulatory focus theory (Higgins, 1997), which asserts that people use more cautious strategies when attempting to prevent negative outcomes, and less cautious, more eager strategies when pursuing positive outcomes.

On average, people set higher thresholds under reward than in the neutral condition, and lower thresholds under punishment than in the neutral condition. However, these mean differences mask variability in how people adjust their thresholds in response to incentives. In both the reward and punishment conditions, about the same number of participants raised their threshold (relative to neutral) as lowered their threshold. Moreover, these changes in threshold were positively correlated $(C I=0.56,0.75$; see supporting information), such that participants who raised their threshold under reward also tended to raise their threshold under punishment. Future work is needed to understand the source of these individual differences. However, a main conclusion that can be drawn from this research is that, regardless of whether people raise or lower their threshold when incentivized, people tend to set higher thresholds under reward than under punishment.

Another question is to what extent information processing is sensitive to the magnitude of the reward or punishment. We operationalized reward and punishment by varying the sign of the incentive (people were punished with a loss versus rewarded with a gain). However, we did not vary the magnitude of the incentive, so it remains to be seen how much changes in the size of the gain or loss affect these processes.

A novel aspect of this work is that it takes advantage of a unique feature of the accumulator framework (e.g., Brown \& Heathcote, 2005, 2008; Usher \& Mcclelland, 2001, 2004). Accumulator models such as the LBA assume that separate evidence accumulators exist for each response alternative, each of which is driven by its own rate of evidence accumulation. These models make it possible to disentangle the quality of the information (the difference in rates for each accumulator) from the quantity of information being processed (the sum of the rates). We believe that understanding how task factors might differentially influence processing quality and quantity is a promising avenue for future research.

The main conclusion of this research is that the information an individual extracts from their environment is not entirely determined by the objective properties of the stimulus. On average, punishment reduces the quality and overall quantity of the extracted information. The decision strategies also depend on how performance is incentivized, with people employing less cautious strategies when faced with punishment than when offered a reward. This research highlights how contextual factors in the environment interact with people's higher order goals to influence information processing dynamics (see also Palada, Neal, Tay, \& Heathcote, 2018; Rae, Heathcote, Donkin, Averell, \& Brown, 2014). 


\section{References}

Brehm, J. W., \& Self, E. A. (1989). The intensity of motivation. Annual Review of Psychology, 40, 109-131. Brown, S. D., \& Heathcote, A. (2005). A ballistic model of choice response time. Psychological Review, 112, 117-128. doi: 10.1037/0033-295X.112.1.117

Brown, S. D., \& Heathcote, A. (2008). The simplest complete model of choice response time: Linear ballistic accumulation. Cognitive Psychology, 57, 153-178. doi: 10.1016/j.cogpsych.2007.12.002

Donkin, C., Averell, L., Brown, S., \& Heathcote, A. (2009). Getting more from accuracy and response time data: Methods for fitting the linear ballistic accumulator. Behavior research methods, 41, 1095-110. doi: 10.3758/BRM.41.4.1095

Frank, M. J., Seeberger, L. C., \& O'Reilly, R. C. (2004). By carrot or by stick: Cognitive reinforcement learning in parkinsonism. Science, 306, 1940-1943.

Galea, J. M., Mallia, E., Rothwell, J., \& Diedrichsen, J. (2015). The dissociable effects of punishment and reward on motor learning. Nature Neuroscience, 18, 597-602. doi: 10.1038/nn.3956

Guitart-Masip, M., Huys, Q. J., Fuentemilla, L., Dayan, P., Duzel, E., \& Dolan, R. J. (2012). Go and no-go learning in reward and punishment: Interactions between affect and effect. NeuroImage, 62, 154-166. doi: 10.1016/j.neuroimage.2012.04.024

Higgins, E. T. (1997). Beyond pleasure and pain. American Psychologist, 52, 1280-1300.

Hockey, G. R. J. (1997). Compensatory control in the regulation of human performance under stress and high workload: A cognitive-energetical framework. Biological Psychology, 45, 73-93. doi: 10.1016/ S0301-0511(96)05223-4

Kahneman, D. (1973). Attention and effort. Englewood Cliffs, NJ: Prentice-Hall.

Kahneman, D., \& Tversky, A. (1979). Propsect theory: An analysis of decision under risk. Econometrica, 47, 263-291.

Kanfer, R., \& Ackerman, P. L. (1989). Motivation and cognitive abilities: An integrative/aptitude treatment interaction approach to skill acquisition. Journal of Applied Psychology, 74, 657-690. doi: 10.1037// 0021-9010.74.4.657

Kool, W., \& Botvinick, M. (2014). A labor/leisure tradeoff in cognitive control. Journal of Experimental Psychology: General, 143, 131-141. doi: 10.1037/a0031048

Kruglanski, A. W., Belanger, J. J., Chen, X., Catalina, K., Pierro, A., \& Manetti, L. (2012). The energetics of motivated cognition: A force-field analysis. Psychological Review, 119, 1-20.

Lee, M. D., \& Wagenmakers, E.-J. (2013). Bayesian cognitive modeling: A practical course. New York, NY: Cambridge University Press.

Leknes, S., \& Tracey, I. (2008). A common neurobiology for pain and pleasure. Nature Neuroscience Reviews, 9, 314-320.

O’Doherty, J., Kringlebach, M. L., Rolls, E. T., Hornak, J., \& Andrews, C. (2001). Abstract reward and punishment representations in the human orbitofrontal cortex. Nature Neuroscience, 4, 95-102.

Palada, H., Neal, A., Tay, R., \& Heathcote, A. (2018). Understanding the causes of adapting, and failing to adapt, to time pressure in a complex multistimulus environment. Journal of Experimental Psychology: Applied, 24, 380-399. doi: 10.1037/xap0000176

Palminteri, S., Khamassi, M., Joffily, M., \& Coricelli, G. (2015). Contextual modulation of value signals in reward and punishment learning. Nature Communications, 6, 1-14. doi: 10.1038/ncomms9096

Pilly, P. K., \& Seitz, A. R. (2009). What a difference a parameter makes: A psychophysical comparison of random dot motion algorithms. Vision research, 49, 1599-1612. doi: 10.1016/j.visres.2009.03.019

Rae, B., Heathcote, A., Donkin, C., Averell, L., \& Brown, S. (2014). The Hare and the Tortoise: Emphasizing speed can change the evidence used to make decisions. Journal of Experimental Psychology: Learning, Memory, and Cognition, 40, 1226-1243. doi: 10.1037/a0036801

Ratcliff, R., \& Smith, P. L. (2004). A comparison of sequential sampling models for two-choice reaction time. Psychological Review, 111, 333-367. doi: 10.1037/0033-295X.111.2.333

Sewell, D. K., \& Smith, P. L. (2016). The psychology and psychobiology of simple decisions: Speeded choice and its neural correlates. In M. Reuter \& C. Montag (Eds.), Neuroeconomics: Studies in 
neuroscience, psychology and behavioral economics (pp. 253-292). Berlin, Heidelberg: Springer. doi: 10.1007/978-3-642-35923-1_14

Usher, M., \& Mcclelland, J. L. (2001). The time course of perceptual choice: The leaky, competing accumulator model. Psychological Review, 108, 550-592. doi: 10.1037//0033-295X.108.3.550

Usher, M., \& Mcclelland, J. L. (2004). Loss aversion and inhibition in dynamical models of multialternative choice. Psychological Review, 111, 757-769. doi: 10.1037/0033-295X.111.3.757 